\title{
ASSESSMENT OF DRUG RELATED PROBLEMS IN PATIENTS WITH CHRONIC DISEASES IN THE GENERAL MEDICINE UNITS OF A TERTIARY CARE HOSPITAL
}

\author{
MANNU MERIA WINCENT ${ }^{1}$, D. POTRILINGAM ${ }^{1}$, ANAGHA V. ${ }^{1}$, SAJITH CHACKO JACOB ${ }^{1}$, ANDHUVAN G. ${ }^{1}$ \\ ${ }^{1}$ Department of Pharmacy Practice, PSG College of Pharmacy, Coimbatore, Tamilnadu, India \\ Email: visitandhuvan@yahoo.com
}

Received: 27 Jul 2017 Revised and Accepted: 02 Nov 2017

\begin{abstract}
Objective: Patients with chronic diseases are more prone to develop drug-related problems (DRPs), which can further worsen their quality of life. The aim of this study was to determine factors and medications associated with DRPs in patients with chronic disease.

Methods: This prospective interventional study was conducted for a duration of 6 mo in the in-patients of general medicine department of PSG Hospital, Coimbatore. DRPs were identified, assessed and recorded as per pharmaceutical care network Europe (PCNE) V5.01 criteria. Chi-square and correlation test were used to analyze the data for identifying factors associated with DRPs.

Results: A total of 137 patients were enrolled for the study, of which 66 patients developed DRPs. The most prevalent DRP was found to be drug choice problem. The major causes of DRPs were found to be drug and dose selection. Antidiabetic drugs were found to be more associated with drug-related problems. The incidence of drug-related problems was high in patients aged between 50 to $59 \mathrm{y}$. Association between gender, length of hospital stays and polypharmacy with DRPs was found to be statistically significant. 58.33\% of the total drug-related problems were completely solved and $19.05 \%$ were partially solved.
\end{abstract}

Conclusion: The incidence of DRPs in the General Medicine department of the hospital was high. The use of an appropriate tool such as PCNE may assist pharmacists and other healthcare professionals to systematically identify, categorize and report drug-related problems.

Keywords: Drug-related problems, Chronic diseases, PCNE, Association

(C) 2017 The Authors. Published by Innovare Academic Sciences Pvt Ltd. This is an open access article under the CC BY license (http://creativecommons.org/licenses/by/4.0/) DOI: http://dx.doi.org/10.22159/ijpps.2017v9i12.21660

\section{INTRODUCTION}

As per the headline in pharmacy today, 2001 "drug-related problems (DRPs); once a \$76.6Billion a headache, now a \$177.4 Billion a migraine". DRPs may be defined as "an event or circumstance involving drug therapy that actually or potentially interferes with desired health outcome" [1]. DRPs may occur in any phases of medication usage; it may start from the prescribing phase and may last till the drug dispensing phase [1]. Improper or incomplete history collection and lack of patient follow-ups might also lead to DRPs [1]. It is recognized that several patient populations may be at high risk to DRPs, which includes pediatric population, geriatric population and people with co-morbid conditions. A statistical report from Delhi shows that out of every 1000 prescriptions, approximately 82 are likely to result in an adverse event, indicating the magnitude of the problems in India [2]. Studies also show that the awareness on the need of adverse drug reaction identification and reporting is only moderate among paramedics [3].

Pharmaceutical care network Europe (PCNE) criteria for DRP classification has been critically apprised and is recognized as the most appropriate classification, reflecting most appropriate outcomes with reproducible results [5]. The classification tool was used in many other published studies to assess DRP occurrence, hence this criterion has been used in our study for identification and categorization of problems, their causes, interventions and their outcomes [6].

\section{MATERIALS AND METHODS}

This was a prospective interventional study and was conducted in a multi-speciality hospital in south India. The study was approved by an institutional human ethical committee of PSG IMSR (approval number: 16/057), Coimbatore. The study was conducted over a period of 6 mo (Jan 2016-June 2016), and the sample size was estimated to be 150 . Patients above the age of $18 \mathrm{y}$ with chronic diseases admitted to the general medicine department of the hospital and who were willing to participate in the study were recruited. Informed consent was received from each study participant prior to their participation. Data were collected and recorded in a specially designed data collection form. DRPs were classified, causes for which were analyzed, interventions were proposed and the outcomes were grouped as per PCNE v5.01 criteria. The association between various factors and the occurrence of DRPs was calculated statistically using either chi-square test or Pearsons correlation test followed by an odds ratio to measure the correlation. Results were considered statistically significant if the $p$ value is less than 0.05 . SPSS version 17 was used for statistical calculation.

\section{RESULTS}

\section{Demographic details}

The study was carried out in a total number of 137 patients. Information pertaining to each patient was obtained and recorded. Out of 137 patients, 83 (61\%) were male and 54 (39\%) were female.Among 137 patients enrolled in the study, 28 were smokers, 13 were alcoholics, 14 were both alcoholic and smoker, beetle nut and tobacco chewing habits were found in 4 . Tabulated data of the demographic features of the study population are given in table 1.

Disease distribution in the participants

Out of 137 patients, 132 (41.7\%) had cardiovascular diseases and 75 $(23.73 \%)$ had endocrinological disorders. Among the patients with endocrinological disorders, $70(22.15 \%)$ had diabetes, and $8(2.53 \%)$ had diabetes-related complications. Respiratory disorders, neurological disorders and rheumatic diseases were found in a number of 28 (8.87\%), 20 (6.32\%) and 19 (6\%) patients respectively. Disease distribution in the study population is shown in table 2 . 
Table 1: Demographic details and characteristics of patients

\begin{tabular}{|c|c|c|}
\hline Details & Characteristics & Number (Percentage \%) \\
\hline \multirow[t]{2}{*}{ Gender } & Male & $83(61 \%)$ \\
\hline & Female & $54(39 \%)$ \\
\hline \multirow[t]{3}{*}{ Age } & $20-39 y$ & $15(10.95 \%)$ \\
\hline & 40-59 years & $53(38.7 \%)$ \\
\hline & $\geq 60$ years & $69(50.35 \%)$ \\
\hline \multirow[t]{4}{*}{ Social habits } & Alcoholism & $13(9.35 \%)$ \\
\hline & Smoking & $28(20.14 \%)$ \\
\hline & Alcoholism and smoking & $14(10.07)$ \\
\hline & Others & $4(2.89 \%)$ \\
\hline \multirow[t]{5}{*}{ Number of drugs prescribed } & $\leq 4$ & $15(10.4 \%)$ \\
\hline & $5-7$ & $88(64.23 \%)$ \\
\hline & $8-10$ & $21(15.32 \%)$ \\
\hline & $11-13$ & $9(6.56 \%)$ \\
\hline & $\geq 14$ & $4(2.9 \%)$ \\
\hline \multirow[t]{2}{*}{ Length of hospital stay } & $<5 d$ & $50(36.5 \%)$ \\
\hline & $\geq 5 \mathrm{~d}$ & $87(63.50 \%)$ \\
\hline
\end{tabular}

Table 2: Disease distribution in the study population

\begin{tabular}{lll}
\hline Diseases & Number of patients & Percentage \\
\hline Endocrine disorders & & 3.16 \\
T1DM $^{*}$ & 10 & 18.99 \\
T2DM $^{* *}$ & 60 & 1.58 \\
Others & 5 & 8.87 \\
Respiratory disorders & 28 & 24.68 \\
Cardiovascular disorders & & 17.1 \\
Hypertension & 78 & 6 \\
Others & 54 & 4.75 \\
Rheumatic diseases & 19 & 6.32 \\
Nephrolological disorders & 15 & 8.55 \\
Neurological disorders & 20 & 27 \\
Others & & \\
\hline
\end{tabular}

"T1DM: Type 1 diabetes mellitus, ${ }^{* * T 2 D M: ~ T y p e ~} 2$ diabetes mellitus

\section{Distribution of DRPs in the study population}

Out of 137 patients, 66 (48\%) patients were found to have DRP. Among these 66 patients, a total of 84 DRPs were identified. The identified DRPs were classified based on PCNE V5.01 criteria and is shown in table 3. Based on which 21 (25\%) problems were categorized as adverse drug reaction, $30(35.72 \%)$ as drug choice problem, 2 (2.38\%) as dosing problem, 8 (9.52\%) as drug use problem, $8(9.52 \%)$ as interaction, and $15(17.86 \%)$ problems as 'others'.

Table 3: Classification of DRP as per PCNE

\begin{tabular}{llll}
\hline Code & Problem & Number of problems & Percentage (\%) \\
\hline P1 & Adverse drug reaction & $\mathbf{2 1}$ & $\mathbf{2 5}$ \\
P1.1 & Side effects (non-allergic) & 15 & \\
P1.2 & Side effects (allergic) & 4 & $\mathbf{3 5 . 7 2}$ \\
P1.3 & Toxic effects suffered & $\mathbf{3 0}$ & $\mathbf{2 . 3 8}$ \\
P2 & Drug choice problem & 7 \\
P2.1 & Inappropriate drug & 2 \\
P2.2 & Inappropriate drug form & 4 \\
P2.3 & Inappropriate duplication of active ingredient & 0 \\
P2.4 & Contra-indication for drug & 3 \\
P2.5 & No clear indication for drug use & 14 \\
P2.6 & No drug prescribed but clear indication & $\mathbf{2}$ \\
P3 & Dosing problem & 0 \\
P3.1 & Drug dose too low & 1 \\
P3.2 & Drug dose too high & 0 \\
P3.3 & Duration of treatment too short & 1 \\
P3.4 & Duration of treatment too long & $\mathbf{8}$ \\
P4 & Drug use problem & 6 \\
P4.1 & Drug not taken/administered & 2 \\
P4.2 & Wrong drug taken/administered & $\mathbf{8}$ \\
P5 & Manifested interactions & $\mathbf{1 5}$ \\
P6 & Others & 3 \\
P6.1 & Patient dissatisfied with therapy & 5 \\
P6.2 & Insufficient awareness of health and disease & 4 \\
P6.3 & Unclear complaints & 3 & $\mathbf{9 . 5 2}$ \\
P6.4 & Therapy failure & $\mathbf{9 . 5 2}$ \\
\hline
\end{tabular}




\section{Drugs involved in DRPs}

Out of the 84 drug-related problems identified in 66 patients, the drug class that was most involved in causing DRP was found to be antidiabetic agents $(n=15)$, followed by cardiovascular drugs $(n=11)$ and anticoagulants $(n=9)$. Other drugs that caused DRPs were steroids, tricyclic antidepressants, antihistamines, analgesics and antibiotics.

\section{Risk factors and their association with DRPs}

Various factors and their association with DRPs were identified. The factors taken into consideration were age, gender, length of hospital stay, number of drugs prescribed and social habits. Among which length of hospital stay, number of drugs prescribed and social habits were found to have a statistically significant association with DRP.
The factors found to be significantly associated with DRPs, along with their 'p' values are shown in table 4.

The incidence of DRPs was high $(28.35 \%)$ in patient aged between 50 to $59 \mathrm{y}$, followed by $28.35 \%$ in the age group of 60 to 69 years, and $17.91 \%$ in the age group of 70 to $79 \mathrm{y}$. The ' $\mathrm{p}$ ' value of significance between age and drug-related problems were found to be 0.153 at $95 \%$ level of significance, hence a statistical significance could not be established. Out of 66 patients, $67 \%$ of DRPs were found in males and $33 \%$ in females. Chi-square test was used to analyze the degree of association between gender and DRP. A pvalue of 0.364 was obtained implicating no association between gender and DRPs. Length of hospital stay showed a significant association with a p-value of 0.018 . Fig. 1 and fig. 2 illustrates the association between length of hospital stay and number of DRPs.

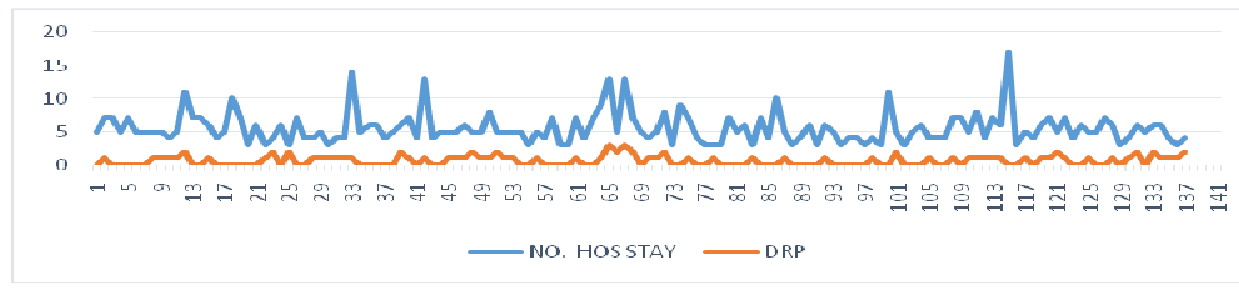

Fig. 1: Correlation between length of hospital stay and incidence of DRP

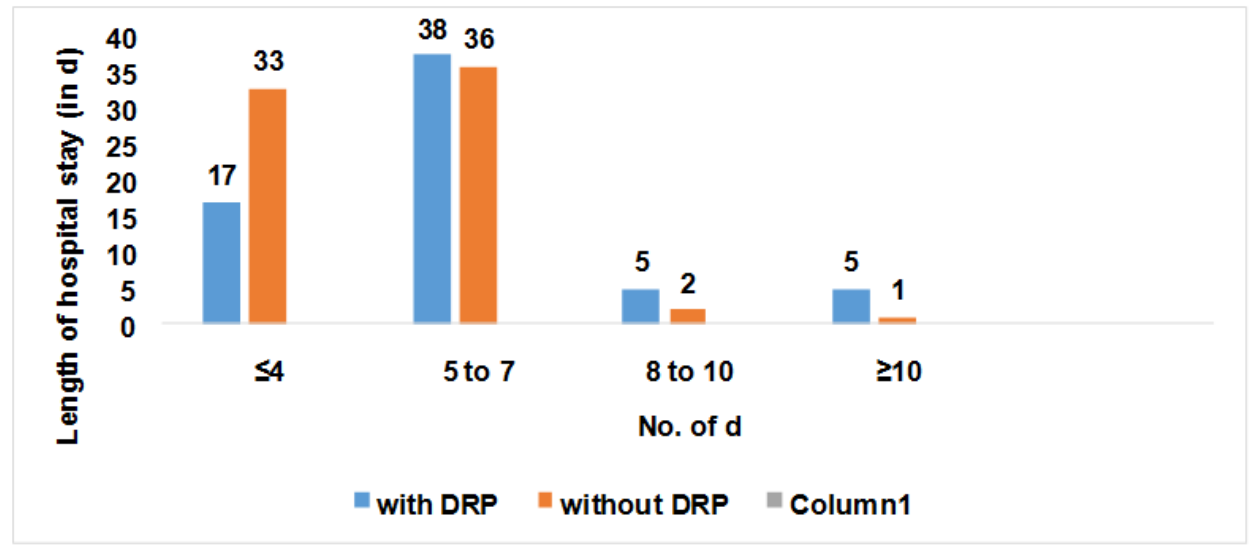

Fig. 2: DRP and hospital stay

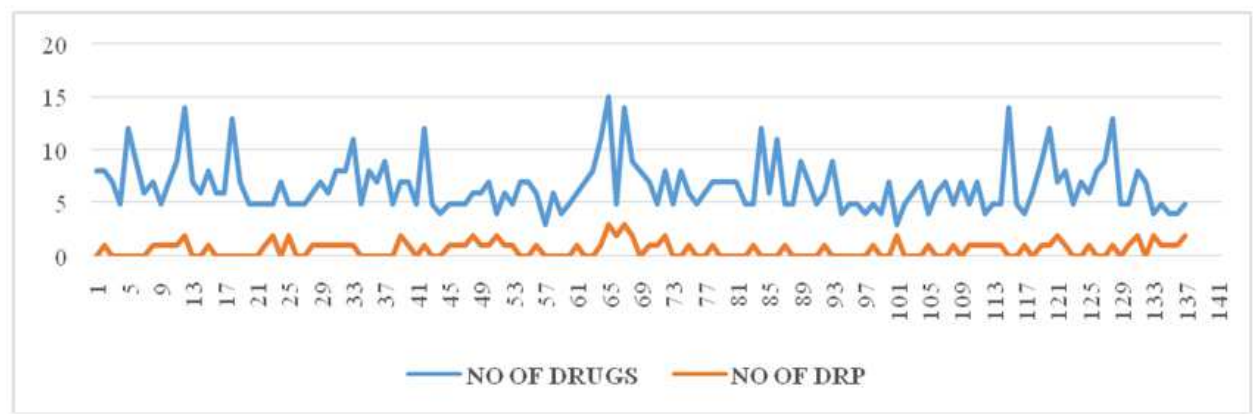

Fig. 3: Correlation between number of drugs prescribed and incidence of DRP

A number of drugs prescribed in the hospital was also found to have statistically significant association with the occurrence of DRP with a p-value of 0.006. Fig. 3 illustrates the association.

Also, social habits like smoking, alcoholism, tobacco and betel nut chewing were found to have a significant association with DRP with a p-value of 0.023 . Odds ratio was calculated to measure the association between social habits and DRPs and a value of 2.217 was obtained implicating that patients with social habits were twice at risk to DRP when compared to another group.
All the factors observed to have a significant association with DRPs are shown in table 4. Correlation between the length of hospital stay and number of drugs prescribed was also estimated in the study population using pearson correlation test (table 5).

The test result showed significant correlation with a p-value of 0.006 , from which it can be concluded that, as the length of hospital stay of the patient increases, the total number of drugs prescribed to the patient increases, which may, in turn, increase the risk of DRPs (fig. 4) 
Table 4: Factors significantly associated with DRP

\begin{tabular}{|c|c|c|c|c|}
\hline Factor & With DRP & Without DRP & Statistical test & P value \\
\hline \multicolumn{5}{|c|}{ Length of hospital stay } \\
\hline$<5 \mathrm{~d}$ & 17 & 33 & Pearson correlation & .018 \\
\hline$\geq$ 5days & 49 & 38 & .201 & \\
\hline \multicolumn{5}{|c|}{ Number of drugs prescribed } \\
\hline$\leq 5$ & 27 & 49 & Person correlation. & .006 \\
\hline$>5$ & 39 & 22 & 305 & \\
\hline Social habits & & & & .023 \\
\hline Yes & 34 & 23 & Pearson Chi square & \\
\hline No & 32 & 48 & 5.147 & \\
\hline
\end{tabular}

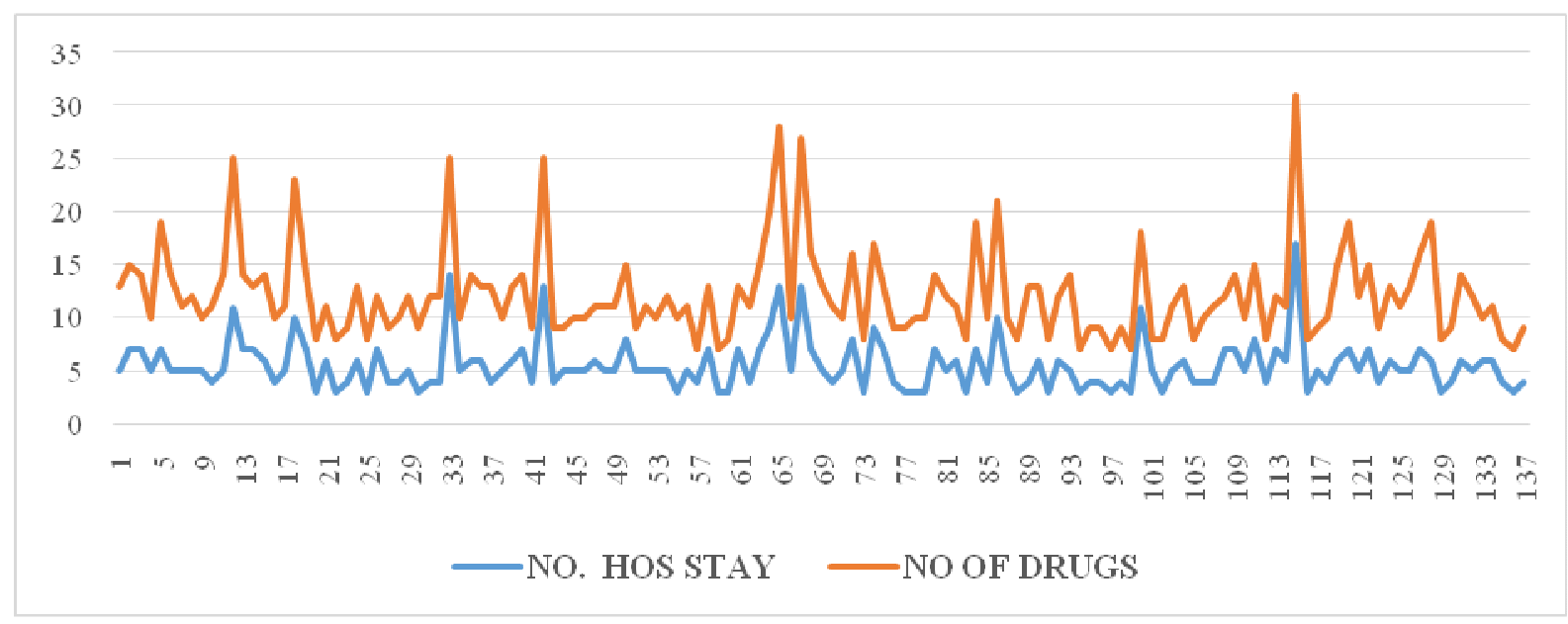

Fig. 4: Correlation between length of hospital stay and number of drugs prescribed

Table 5: Correlation between length of hospital stay and number of drugs prescribed

\begin{tabular}{lll}
\hline Factors & Pearson correlation & P value \\
\hline Length of hospital stay and number of drugs prescribed & .710 & 0.006 \\
\hline
\end{tabular}

Causes for each DRP were also found with the aid of PCNE v5.01 criteria. Causality assessment revealed that improper drug or dose selection was the major cause of DRPs (33.34\%) followed by improper information channelling (24.4\%). The observations corresponding to causality assessment is tabulated in table 6 .
Interventions were also made in regard to each cause of DRP. Interventions were put forward at various levels, namely patient level, prescriber level, drug level and others. Interventions put forward at prescriber level were higher (54.72\%) followed by drug level $(20.75 \%)$. The results are shown in tabulated form in table 7.

Table 6: Causes of DRP as per PCNE

\begin{tabular}{llll}
\hline Code & Cause & Total number & Percentage \\
\hline C1 & Drug/dose selection & $\mathbf{3 0}$ & \\
C1.1 & Inappropriate drug selection & 5 & 3 \\
C1.2 & Inappropriate dosage selection & 1 & \\
C1.3 & More cost effective drug available & 5 & \\
C1.4 & Pharmacokinetic problems & 3 & \\
C1.5 & Synergestic/preventive drug required and not given & 0 & \\
C1.6 & Deterioration or improvement of disease state & 2 & $\mathbf{1 1 . 1 1}$ \\
C1.7 & New symptom or indication revealed/presented & 11 & \\
C1.8 & Manifest side effect, no other cause & $\mathbf{1 0}$ & \\
C2 & Drug use process & 2 & \\
C2.1 & Inappropriate timing of administration & 0 & \\
C2.2 & Drug underused/under-administered & 6 & \\
C2.3 & Drug overused/over-administered & 1 & \\
C2.4 & Therapeutic dug level not monitored & 0 & \\
C2.5 & Drug abused & 1 \\
C2.6 & Patient unable to use drug as directed & $\mathbf{2 2}$ \\
C3 & Information & 3 \\
C3.1 & Instruction for use not known & 0 \\
C3.2 & Patient unaware of reason for treatment & 0 & \\
C3.3 & Patient has difficulties reading or understanding PIL & $\mathbf{2 4 . 4 4}$ \\
C3.4 & Patient unable to understand local language & 4 & \\
\hline
\end{tabular}




\begin{tabular}{lll}
\hline C3.5 & Lack of communication between healthcare professionals/patients & 15 \\
C4 & Patient/Psychological & $\mathbf{1 1}$ \\
C4.1 & Patient forgets to take drug & 0 \\
C4.2 & Patients have concerns with drugs & $\mathbf{1 2 . 2 2}$ \\
C4.3 & Patient suspects side-effects & 1 \\
C4.4 & Patient unwilling to carry financial costs & 5 \\
C4.5 & Patient unwilling to bother physician & 0 \\
C4.6 & Patient unwilling to change drugs & 2 \\
C4.7 & Patient unwilling to adapt lifestyle & 0 \\
C4.8 & Burden of therapy & 1 \\
C4.9 & Treatment not in line with health beliefs & 0 \\
C4.10 & Patient takes food that interacts with drugs & 0 \\
C5 & Logistics & $\mathbf{6}$ \\
C5.1 & Prescribed drug not available (anymore) & 0 \\
C5.2 & Prescribing error (only in case of slip of the pen) & \\
C5.3 & Dispensing error (wrong drug or dose dispensed) & 5 \\
C6 & Others & $\mathbf{6 . 6 7}$ \\
C6.1 & Other cause; specify & 1 \\
C6.2 & No obvious cause & $\mathbf{1 1}$ \\
\hline
\end{tabular}

Table 7: Interventions of DRPs as per PCNE

\begin{tabular}{|c|c|c|c|}
\hline Code & Intervention & Total number & Percentage \\
\hline I0 & No intervention & 3 & 2.83 \\
\hline I1 & At prescriber level & 58 & 54.72 \\
\hline I1.1 & Prescriber informed only & 18 & \\
\hline $\mathrm{I} 1.2$ & Prescriber asked for information & 7 & \\
\hline $\mathrm{I} 1.3$ & Intervention proposed, approved by prescriber & 28 & \\
\hline $\mathrm{I} 1.4$ & Intervention proposed, not approved by prescriber & 4 & \\
\hline $\mathrm{I} 1.5$ & Intervention proposed, outcome unknown & 1 & \\
\hline I2 & At patient/career level & 12 & 11.32 \\
\hline $\mathrm{I} 2.1$ & Patient (medication) counseling & 4 & \\
\hline $\mathrm{I} 2.2$ & Written information provided only & 0 & \\
\hline $\mathrm{I} 2.3$ & Patient referred to prescriber & 1 & \\
\hline $\mathrm{I} 2.4$ & Spoken to family member/caregiver & 7 & \\
\hline I3 & At drug level & 22 & 20.75 \\
\hline $\mathrm{I} 3.1$ & Drug changed to. & 5 & \\
\hline $\mathrm{I} 3.2$ & Dosage changed to.... & 4 & \\
\hline $\mathrm{I} 3.3$ & Formulation changed to.... & 0 & \\
\hline $\mathrm{I} 3.4$ & Instructions for use changed to.... & 1 & \\
\hline I3.5 & Drug stopped & 6 & \\
\hline I3.6 & New drug started & 6 & \\
\hline I4 & Other intervention or activity & 11 & 10.38 \\
\hline I4.1 & Other intervention (specify) & 3 & \\
\hline $\mathrm{I} 4.2$ & Side effect reported to authorities & 6 & \\
\hline
\end{tabular}

Final outcomes of the proposed interventions were also evaluated and it was found that $64.28 \%$ of drug-related problems were completely resolved and $19.05 \%$ were partially resolved.

Table 8: Outcomes of proposed intervention

\begin{tabular}{|c|c|c|c|}
\hline Code & Outcome of intervention & Total number & Percentage \\
\hline 00 & Not known & 0 & 0 \\
\hline 01 & Problem totally solved & 54 & 64.28 \\
\hline 02 & Problem partially solved & 16 & 19.05 \\
\hline 03 & Not solved & 14 & 16.67 \\
\hline 03.1 & Problem not solved, lack of cooperation of patient & 2 & \\
\hline 03.2 & Problem not solved, lack of cooperation of prescriber & 5 & \\
\hline 03.3 & Problem not solved, intervention not effective & 1 & \\
\hline 03.4 & No need or possibility to solve problem & 6 & \\
\hline
\end{tabular}

\section{DISCUSSION}

This study is an estimation of the incidence of DRPs in the general medicine unit since the department chosen was general medicine, the distribution of disease conditions reflects the distribution in the local population of the area. In the study population, most prevalent disease conditions were hypertension (24.68\%) and diabetes mellitus (22.15\%).

Results of studies conducted by the World Health Organization and Central Disease Control and Prevention in India draws a similar conclusion that the statistical prevalence of cardiovascular diseases and diabetes is the highest [6]. Studies also point out that diabetes is the most common secondary cause of kidney diseases [7].

Out of 137 patients recruited for the study, 66 patients were found to have DRPs. A total of 84 were identified in the 66 patients, implicating multiple DRPs in a single patient. PCNE criterion was used to categorize DRPs. This criterion has been critically appraised as the most appropriate classification that reflects the outcomes most appropriately and results reproducibly. Out of 66 patients, $67 \%$ of males and $33 \%$ of females were found to have DRPs. The result showed a male predominance in DRP, which might be due to various risk factors like smoking, alcoholism, sedentary 
lifestyle, etc, compared to the female population. This result is analogous to the study carried out by Sarfaraz Mohammed et al., which showed male predominance over females [8]. This result is also similar to the study conducted by the Ganachari et al., which showed male predominance over females [9]. Similar results have also been obtained in studies conducted by Alagiriswami et al., and Sathishkumar et al., which showed an increase in number of DRPs in male population when compared to females [10]. But a statistical significance for the association could not be established. Till date, there is a lack of evidence to suggest that biological factors associated with gender may affect the pharmacological treatment.

In this study, the most prominent DRP was found to be drug choice problem (35.72\%), followed by adverse drug reaction (ADR) (25\%) and then 'other' problems (17.86\%), followed by interactions and drug use problems with $9.52 \%$ each. This is in contrast with the study conducted by Mahesh Kumar VP et al., which showed that drug interaction was the most prevalent followed by drug choice problems, ADR and 'others' [11].

Different health care settings, difference in sample size, study tool and study population might have resulted in the contrasting results. Hypoglycemia due to insulin and oral hypoglycemic agents in diabetic patients was the most common ADR observed in our study. The same observation was found in another study done in south India by Jaavedh Shareef et al. insulin dosing errors was also highly prevalent in the study population [12].

The incidence of DRPs was high $(28.35 \%)$ in patient aged between 50 to $59 \mathrm{y}$. The study conducted by Jaavedh Shareef et al., endorses this finding [12]. His study says that incidence of DRP is high in the age group of 41-60years. A higher incidence of DRP in this age group might be due to the presence of multiple co-morbid conditions in the age group. The ' $\mathrm{p}$ ' value of significance between age and drugrelated problems were found to be 0.153 at $95 \%$ level of significance, hence a statistical significance could not be established.

Association between the length of hospital stay and DRP was checked. 49 DRPs were found in a patient hospitalized for more than $5 \mathrm{~d}$, and 17 DRPs in those with less than $5 \mathrm{~d}$ of hospital stay. A statistically significant result was obtained in the test for association with a ' $p$ ' value of 0.018 at $95 \%$ confidence interval. This finding was similar to the study done by Mahesh Kumar V. P et al., which showed that length of hospital stay is significantly associated with DRP, at the level of 0.05 [11].

The mean number of drugs prescribed per patient was found to be 6.6. A total of 122 patients were prescribed with 5 or more drugs. Statistical association between the number of drugs prescribed and DRP was found to be significant with a 'p' value of 0.0011 . This result was in accordance with the study carried out by Mahesh Kumar VP et al. which showed that polypharmacy has a statistically significant association at the $\mathrm{p}<0.05$ [11].

National survey conducted in the year 2002 indicate that $50 \%$ of the population taking 5 or more drugs developed DRP [13].

The patient's length of hospital stay and the number of drugs prescribed was correlated, which is found to be significant at 0.01 level, from which it can be concluded that, as the length of hospital stay of the patient increases, the total number of drugs prescribed to the patient increases, which may increase the risk of DRPs.

The social habits of the patient population were also documented. Among 137 patients, 28 (20.14\%) were smokers, 13(9.35\%) were alcoholics, $14(10.07 \%)$ were both smoker and alcoholic, and 4 $(2.89 \%)$ had habits of betel nut and tobacco chewing. Out of patients with social habits, $34 \%$ of them experienced DRPs. The association between social habits and DRPs was found out to be statistically significant at the $\mathrm{p}$ value of 0.0369 . The possible reason for the significant association between the social habits and the occurrence of DRP might be change in pharmacokinetic parameters in people with social habits like smoking, alcoholism etc.

The major cause of DRP was found to be drug and dose selection (33.34\%), followed by improper information gathering from the patient (24.44\%). This is in accordance with the study conducted by Mahesh Kumar VP et al,, in which drug/dose selection was found to be the major cause for DRP at the rate of $48.8 \%$, followed by patient-related causes. Missing information in medication history collection was the most notable error in our study. Lack of time of physicians, patient inhibition in communicating with physician and language barriers were found to be the major contributing factors for it.

Interventions suggested for the problems were put forward at three levels-prescriber level, patient level and drug level. Out of which most of the interventions were proposed at prescriber level (54.72\%), followed by drug level (20.75\%). This is in contrast to the study conducted by Mahesh Kumar VP, in which the interventions at patient level were the highest followed by prescriber level and then drug level. $58.33 \%$ of the total DRPs were completely solved and $19.05 \%$ were partially solved. A similar study was done in Pakistan showing that a large majority of problems (85\%) were completely solved by pharmacist interventions. A wide difference in the realtime clinical practice setting, when compared to the theoretical knowledge, was the main challenge faced while doing the study. Thus, the study indicates that pharmacist interventions can be an aid in reducing DRPs in hospital settings.

\section{CONCLUSION}

The study shows that the incidence of DRPs within the general medicine unit of the hospital is high, nearly one-half of the inpatients developed drug-related problems. The study also overemphasizes that the incidence of DRPs increases with an increase in the length of hospital stay and number of drugs prescribed. Presence of deleterious social habits is also an important contributing factor to the occurrence of drug-related problems.

\section{LIMITATIONS OF THE STUDY}

The major limitation of the study is that the pediatric population (age $<18$ y) were not included given that fact that even paediatric population are prone to DRPs and also while documentation of DRPs the unmanifested errors in patients could not be covered.

\section{AUTHOR CONTRIBUTION}

Mrs. G. Andhuvan (project guide), was involved in developing the concept and study design. She played a major role in the statistical analysis and result interpretation and is also maintaining patients file and master file of the project.

Ms. Mannu Meria Wincent, Mr. D. Potrilingam, Ms. Anagha. V, Mr. Sajith Chacko Jacob

were involved in developing the study concept, design, screening of patients, informed consent, selection and recruitment of patients, patient evaluation, serious adverse event evaluation and reporting, data collection and monitoring of data, interpretation of data, statistical analysis and interpretation, drafting final report and submission of final report to institutional human ethical committee.

\section{CONFLICT OF INTERESTS}

\section{Declared none}

\section{REFERENCES}

1. Adusumilli PK, Adepu R. Drug-related problems: an overview of various classification systems. Asian J Pharm Clin Res 2014;7:7-10.

2. Pankaj Agarwal, Ajay Sachan, Rajeev K Singla, Pankaj Jain. Statistical analysis of medication errors in Delhi, India. Indo Global J Pharm Sci 2012;2:88-97.

3. Shalini Sivadasan, Mohan Sellapan. A study on the awareness and attitude towards pharmacovigilance and adverse drug reaction reporting among nursing students in a private university, Malaysia. Int J Curr Pharm Res 2015;7:84-9.

4. Hasniza Zaman Huri, Lee Chari Ling. Drug-related problems in type 2 diabetes mellitus patients with dyslipidemia. Bio Med Central 2013;13:1192.

5. Pharmaceutical Care Network Europe Foundation. PCNE classification for drug-related problems version 5.01; 2006.

6. http://www.pcne.org/sig/drp/documents/PCNE\%20classifica tion\%20V5.01.pdf

7. India: Towards Universal Health Coverage 3, chronic diseases and injuries in India. Available from: http://www.who.int/ 
macrohealth/action/NCMH_Burden\%2520of\%2520disease_(2 $9 \% 2520$ Sep\%25202005).pdf. [Last accessed on 20 Jun 2017]

8. Harikesh Maurya, Tirath Kumar. A review on comprehensive overview in the management of nephritic disorders. J Crit Rev 2016;3:34-43.

9. Sarfaraz Mohammed, Sanjay Poudel, Fernance Laloo, Arjun Madhur, Rinson Robert, Binu Mathew. Assessment of drug related problems in a tertiary care teaching hospital, India. Asian J Pharm Clin Res 2017;10:310-3.

10. Ganachari MS, Mahendra Kumar BJ, Shashikala C Wali, Fibin M. Assessment of drug therapy interventions by a clinical pharmacist in a tertiary care hospital. Indian J Pharm Practice 2010;3:22-8.
11. Bhupathy Alagiriswami, Madhan Ramesh, Gurumurthy Parthasarathi, Hatthur Basavanagowdappa. A study of clinical pharmacist-initiated changes in drug therapy in a teaching hospital. Indian J Pharm Practice 2009;1:36-45.

12. Maheshkumar VP, Dhanapal CK, Ramakrishna Rao M. Outcomes of clinical pharmacist's interventions in solving drug-related problems in geriatric patients of a rural teaching hospital. Pharma Innovation J 2016;5:99-105.

13. Javedh Shareef, Sandeep B, CS Shastry. Assessment of drugrelated problems in a patient with cardiovascular diseases in a tertiary care teaching hospital. J Pharm Care 2014;2:70-6.

14. Sujit Rambhade, Anup Chakarborty, Anand Shrivastava, Umesh K. Patil and Ashish Rambhade. A survey on polypharmacy and use of inappropriate medications. Toxicol Int 2012;19:68-73. 